\title{
PATRONES DE ENDEMISMO Y DISYUNCIÓN DE LOS GÉNEROS DE EUPHORBIACEAE SENSU LATO: UN ANÁLISIS PANBIOGEOGRÁFICO
}

\author{
Martha MartíneZ-Gordillo ${ }^{1}$ y JUAN J. MORRONE ${ }^{2}$ \\ ${ }^{1}$ Herbario "FCME", Departamento de Biología Comparada, Facultad de Ciencias, Universidad Nacional \\ Autónoma de México. Apdo. Postal 70-181, México 04510, D.F., México. \\ Correo-e: mjmg@hp.fciencias.unam.mx. \\ ${ }^{2}$ Museo de Zoología "Alfonso L. Herrera", Departamento de Biología Evolutiva, Facultad de Ciencias, \\ Universidad Nacional Autónoma de México. Apdo. Postal 70-399, México 04510, D.F., México \\ Correo-e: jjm@hp.fciencias.unam.mx.
}

\begin{abstract}
Resumen: Se analizaron los patrones de distribución de los géneros de Euphorbiaceae bajo un enfoque panbiogeográfico, empleando el método del análisis de parsimonia de endemismos (PAE). Se obtuvieron cuatro trazos generalizados, que unen las regiones siguientes: (1) Neotropical-Afrotropical (determinado por los géneros Amanoa, Caperonia, Conceveiba, Manprounea, Pogonophora, Savia y Tetrorchidium); (2) Australiana Templada-Australiana Tropical-Neoguineana-Oriental (determinado por los géneros Actephila, Baloghia, Choriceras, Petalostigma y Sauropus); (3) Australiana Templada-Australiana TropicalNeoguineana-Afrotropical-Neotropical (determinado por los géneros Acalypha, Alchornea, Cleidion, Drypetes, Margaritaria, Microstachys, Omphalea y Phyllanthus); y (4) Neoguineana-Oriental-Afrotopical (determinado por los géneros Glochidion, Macaranga, Microdesmis y Shirakopsis). Dos trazos generalizados se superponen en la región Afrotropical, la cual es identificada como un nodo.

Palabras clave: biogeografía, distribución, endemismo, Euphorbiaceae s. l., PAE, trazos generalizados.

Abstract: Distributional patterns of the genera of Euphorbiaceae were analyzed under a panbiogeographic approach, using the parsimony analysis of endemicity (PAE) method. Four generalized tracks were obtained, joining the following regions: (1) Neotropical-Afrotropical (determined by the genera Amanoa, Caperonia, Conceveiba, Manprounea, Pogonophora, Savia and Tetrorchidium); (2) Temperate Australian-Tropical Australian-Neoguinean-Oriental (determined by the genera Actephila, Baloghia, Choriceras, Petalostigma and Sauropus); (3) Temperate Australian-Tropical Australian-Neoguinean-AfrotropicalNeotropical (determined by the genera Acalypha, Alchornea, Cleidion, Drypetes, Margaritaria, Microstachys, Omphalea and Phyllanthus); and (4) Neoguinean-Oriental-Afrotopical (determined by the genera Glochidion, Macaranga, Microdesmis and Shirakopsis). Two generalized tracks overlap in the Afrotropical region, which is identified as a node.
\end{abstract}

Key words: biogeography, distribution, endemism, Euphorbiaceae $s . l$, generalized tracks, PAE.

$\mathbf{L}$ as Euphorbiaceae poseen distribución cosmopolita, con excepción de las zonas polares. Sus especies se encuentran principalmente en las regiones tropicales y subtropicales (Radcliffe-Smith, 1987). Constan de cinco subfamilias, 49 tribus, entre 317 y 339 géneros (Webster, 1994; Radcliffe-Smith, 2001) y cerca de 8,100 especies (Mabberley, 1998). Webster (1994) y Radcliffe-Smith (2001) clasifican a las Euphorbiaceae en cinco subfamilias: Phyllanthoideae, Oldfieldioideae, Acalyphoideae,
Crotonoideae y Euphorbioideae; las dos primeras poseen dos óvulos por lóculo, mientras que las tres últimas presentan uno solo. La monofilia de las Euphorbiaceae no ha sido confirmada en análisis filogenéticos basados en secuencias de DNA (Savolainen et al., 2000; Soltis et al., 2000; APGII, 2003), por lo que se ha propuesto que sus especies en realidad pertenecerían a tres familias diferentes (APGII, 2003): Euphorbiaceae sensu stricto, integrada por las tres subfamilias uniovuladas (Acalyphoideae, Crotonoideae y 
Euphorbioideae); Phyllanthaceae, que corresponde a la subfamilia Phyllanthoideae, con especies biovuladas y polen no espinoso; y Picrodendraceae, que corresponde a la subfamilia Olfieldioideae, con especies biovuladas y polen espinoso. Por otro lado, los géneros Drypetes, Lingelsheimia, Putranjiva y Sibangea, incluidos en las clasificaciones tradicionales dentro de las Phyllanthoideae, están considerados ahora dentro de una familia distinta: Putranjivaceae, con base en la presencia de glucosinolatos. Si bien la monofilia de las Euphorbiaceae sensu lato es dudosa, cabría aclarar que los análisis panbiogeográficos no exigen que los taxones analizados constituyan un grupo monofilético, siendo incluso deseable llevar a cabo los análisis de este tipo con taxones no relacionados entre sí (Morrone, 2004). Más aún, creemos que un análisis de este tipo quizá podría brindar alguna clave para comprender la sistemática de estos taxones.

Los análisis de los patrones de distribución global de la familia son escasos. Bentham (1878) discute el origen de la familia, ubicando a los taxones ancestrales en el Viejo Mundo. Pax (1910) estudia la distribución de la tribu Jatrophae. Croizat (1972) y Leach (1976) analizan la tribu Euphorbieae. Webster (1994) discute algunos aspectos de la distribución de las subfamilias, destacando la importancia de algunos géneros endémicos y disyuntos. De acuerdo con este autor, África y Madagascar son las regiones donde se distribuyen los taxones más plesiomórficos de las Phyllantoideae; América del Sur es la región de la diferenciación inicial de las Crotonoideae y Ofieldieiodeae; y América del Sur y África son las áreas donde se diversificaron las Acalyphoideae y Euphorbideae.

El método panbiogeográfico fue propuesto inicialmente por Croizat $(1958,1964)$ para representar objetivamente la distribución geográfica de los taxones, lo que se hace uniendo sus localidades de distribución mediante líneas, llamadas trazos individuales, en los que la distancia entre ellas es la mínima (Craw et al., 1999). La manera más frecuente de orientar los trazos es a partir de una línea de base, la cual se designa de acuerdo con los rasgos geográficos/ geológicos de mayor relevancia, como cuencas oceánicas. Sin embargo, las dificultades asociadas con la identificación de líneas de base (u otros criterios de orientación, como centros de masas o información filogenética) hacen que sea aconsejable trabajar con trazos no orientados (Morrone, 2004). Los trazos individuales de diferentes taxones que coinciden determinan trazos generalizados y éstos indican componentes bióticos ancestrales, que fueron fragmentados por eventos tectónicos o climáticos (Morrone y Crisci, 1995; Morrone, 2004). Las áreas donde se unen trazos generalizados diferentes se denominan nodos y representan sitios con alta riqueza taxonómica y de relaciones geográficas y filogenéticas (Morrone y Crisci, 1995).

El análisis de parsimonia de endemismos (PAE) permite construir cladogramas a partir de matrices de presencia- ausencia de especies y taxones supraespecíficos (Rosen, 1988; Morrone, 1994a, b, 1998, 2004; Escalante y Morrone, 2003). Algunos autores (Craw et al., 1999; Luna Vega et al., 2000; Morrone y Márquez, 2001; Morrone, 2004) han propuesto utilizar este método para llevar a cabo análisis panbiogeográficos, donde los clados sustentados por dos o más taxones son considerados equivalentes a trazos generalizados. De acuerdo con lo propuesto por LunaVega et al. (1999) y García-Barros et al. (2002), una vez que se obtienen los cladogramas más parsimoniosos, se "desconectan" los taxones que sustentan los diferentes clados y se analiza la matriz nuevamente para buscar clados sustentados por otros. Los pasos seguidos en el análisis de parsimonia de endemismos (Morrone, 2004) son los siguientes: (1) construir trazos individuales para taxones diferentes, conectando las localidades donde se distribuyen mediante un árbol de tendido mínimo; (2) construir una matriz de áreas por trazos individuales, en la que la presencia de un taxón en un área se representa con un "1" y la ausencia con un "0", añadiendo un área "externa" con todos " 0 ", con el objeto de enraizar el cladograma; (3) analizar la matriz con un algoritmo de parsimonia y conectar las áreas incluidas en cada clado sustentado por al menos dos trazos individuales como parte de un mismo trazo generalizado; (4) "desconectar" los trazos individuales que sustentan los clados obtenidos y analizar nuevamente la matriz para buscar clados sustentados por otros; y (5) indicar en un mapa los trazos generalizados y (si existen) las líneas de base y los nodos.

Los patrones de endemismo y distribución disyunta de los géneros de Euphorbiaceae son importantes para entender su historia evolutiva. Nuestro objetivo fue analizar la distribución de los géneros de la familia, aplicando los métodos de la panbiogeografía, buscando identificar los patrones generalizados de su distribución.

\section{Material y métodos}

Los datos de distribución se obtuvieron a partir de estudios de revisión de Müller Argoviensis (1865, 1866), Bentham (1878), Pax (1890), Ferguson (1901), Millspaugh (1916), Standley (1923), Pax y Hoffman (1924, 1931), Leandri (1939, 1969, 1971, 1972a,b), Croizat (1940, 1941, 1944, 1945), Lundell (1940), Standley y Steyermark (1949), Johnston (1959), McVaugh (1961), Johnston y Warnock (1962), Airy Shaw (1971, 1980a,b, 1982), Borhidi y Muñiz (1977), Radcliffe-Smith (1987, 2001), Webster (1987, 1993, 1994), Martínez (1996) y Govaerts et al. (2000). También se revisaron ejemplares depositados en las colecciones MEXU, ENCB, MO, XAL y CHAPA. Los 339 géneros analizados (apéndice 1) son los propuestos por Radcliffe-Smith (2001) quien, a diferencia de Webster (1994), considera a géneros como Crotonopsis, Eremocarpus y Julocroton, entre otros, como diferentes. 
Se consideraron como unidades del estudio las regiones biogeográficas de Morrone (2002). Cada uno de los géneros de Euphorbiaceae s.l. se consideró como un carácter. Se construyó una matriz de 12 regiones $\times 339$ géneros, 208 de los cuales no son informativos a este nivel porque se trata de géneros endémicos de una región. Los análisis se hicieron usando Nona 2.0 (Goloboff, 1993) y WinClada 1.00.08 (Nixon, 2002). Se incluyeron todos los caracteres dentro del análisis aunque en realidad sólo 139 fueron informativos. Las topologías se orientaron usando como raíz a un área codificada con ceros. Se construyó un cladograma de consenso estricto. Finalmente, los clados sustentados por dos o más géneros fueron representados en un mapa como trazos generalizados y en la zona de superposición de dos trazos generalizados se ubicó el nodo.

Diversidad y endemismo de Euphorbiaceae sensu lato. La riqueza de géneros de Euphorbiaceae sensu lato se presenta en el Cuadro 1, analizando cada subfamilia de acuerdo con las regiones propuestas por Morrone (2002).

La subfamilia Phyllanthoideae tiene 10 tribus y 60 géneros (Radcliffe-Smith, 2001); de estos últimos 58.3\% es endémico. La región Afrotropical tiene 19 géneros endémicos (Apodiscus, Blotia, Centroplacus, Keayodendron, Leptonema, Lingelsheimia, Martretia, Maesobotrya, Pentabrachion, Petalodiscus, Protomegabaria, Pseudolachnostylis, Sibangea, Spondianthus, Thecacoris, Uapaca, Wielandia, Zimmermannia y Zimmermanniopsis), seguida de la región Neotropical con 12 (Astrocasia, Celianella, Chascotheca, Chonocentrum, Didymocistus, Discocarpus, Gonatogyne, Hieronyma, Jablonskia, Phyllanoa, Richeria y Tacarcuna). Entre los géneros endémicos, los más diversos son Uapaca (61 especies) y Maesobotrya (20). Esta subfamilia posee 2,110 especies, con el mayor centro de diversidad genérica en la región Afrotropical (61\%). Algunos géneros, como Amanoa, Drypetes, Margaritaria, Meineckia y Phyllanthus, tienen distribución disyunta en América del Sur y África (Webster, 1994). Los géneros más diversos son Phyllanthus (800 especies), Glochidion (300), Antidesma (170) y Drypetes (200). Ejemplos de distribución disyunta son los géneros Amanoa (figura 1a), Actephila (figura 1b), Bridelia (figura 3), Cleistanthus (figura 2) y Meineckia (figura 4a).

La subfamilia Olfieldioideae tiene cuatro tribus, 27 géneros y 100 especies (Radcliffe-Smith, 2001) y es la menor de todas. Los centros de mayor diversidad genérica se encuentran en las regiones Neoguineana y Australiana Templada, con nueve géneros cada una. Existe un alto endemismo genérico $(74.1 \%)$, resaltando las regiones Neotropical y Afrotropical, donde todos los géneros son endémicos (seis y cinco, respectivamente). De todos los géneros, 10 son monotípicos. El género más diverso es Austrobuxus (20 especies). Austrobuxus (figura 5b) y Choriceras (figura 4b) son ejemplos de distribuciones disyuntas.

La subfamilia Acalyphoideae tiene 21 tribus, 120 géneros (Radcliffe-Smith, 2001) y 2,584 especies. El centro con mayor diversidad se encuentra en la región Oriental (46.7\% de las especies). Los géneros más diversos son Acalypha (450 especies), Dalechampia (110), Macaranga (280) y Tragia (150). El endemismo genérico es de 50.3\%, con la mayor concentración en las regiones Neotropical y Afrotropical. En la primera son endémicos Acidoton, Adenophaedra, Alchorneopsis, Angostylis, Aparisthmium, Argythamnia, Astrococcus, Caryodendron, Chiropetalum, Ditaxis, Dysopsis, Eleutherostigma, Enriquebeltrania, Gavarretia, Gitara, Haematostemon, Lasiocroton, Leucocroton, Pera, Philyra, Platygyna, Polyandra, Romanoa y Vigia; mientras que en la segunda lo son Amyrea, Argomuellera, Bossera, Cephalocroton, Cephalocrotonopsis, Claoxylopsis, Cordemoya, Crotonogynopsis, Cyttaranthus, Discoclaoxylon, Discoglypremna, Erythrococca, Droceloncia, Lobanilia, Mareya, Mareyopsis, Necepsia, Orfilea, Panda,

Cuadro 1. Número de géneros (gén.) y endemismos (endem.) de las subfamilias de Euphorbiaceae, en las regiones propuestas por Morrone (2002), excluyéndose la región Antártica en la cual no se registran especies de esta familia. AFR: Afrotropical, AND: Andina, AUE: Australiana Templada, AUT: Australiana Tropical, CAP: Capense, NEA: Neártica, NEC: Neozelandesa, NEG: Neoguineana, NEO: Neotropical, ORI: Oriental, PAL: Paleártica.

\begin{tabular}{|c|c|c|c|c|c|c|c|c|c|c|c|c|}
\hline Subfamilias & NEA & PAL & NEO & AFR & ORI & AUT & AND & CAP & NEG & AUE & NEC & $\begin{array}{l}\text { Total } \\
\text { gén./ } \\
\text { endem. }\end{array}$ \\
\hline Phyllanthoideae & $2 / 1$ & $5 / 0$ & $20 / 12$ & $37 / 19$ & $20 / 0$ & $10 / 0$ & $1 / 0$ & $3 / 1$ & $17 / 1$ & $12 / 0$ & $2 / 1$ & $60 / 35$ \\
\hline Oldfieldioideae & $1 / 0$ & 0 & $6 / 6$ & $5 / 5$ & $2 / 1$ & $2 / 0$ & 0 & 1 & $9 / 4$ & $9 / 4$ & 0 & $27 / 20$ \\
\hline Acalyphoideae & $3 / 0$ & $10 / 1$ & $38 / 24$ & $46 / 24$ & $56 / 12$ & $8 / 1$ & $1 / 1$ & $4 / 0$ & $39 / 1$ & $12 / 0$ & 0 & $120 / 64$ \\
\hline Crotonoideae & $3 / 2$ & $5 / 0$ & $23 / 19$ & $27 / 17$ & $23 / 6$ & $7 / 3$ & $1 / 0$ & $2 / 0$ & $22 / 4$ & $8 / 0$ & $1 / 0$ & $74 / 51$ \\
\hline Euphorboideae & $8 / 1$ & $6 / 1$ & $32 / 20$ & $25 / 12$ & $16 / 2$ & $7 / 0$ & $3 / 1$ & $4 / 0$ & $13 / 1$ & $7 / 0$ & $3 / 0$ & $58 / 38$ \\
\hline Totales & $17 / 4$ & $26 / 2$ & $119 / 81$ & $140 / 77$ & $117 / 21$ & $34 / 4$ & $6 / 2$ & $14 / 1$ & $100 / 11$ & $48 / 4$ & $6 / 1$ & $339 / 208$ \\
\hline
\end{tabular}




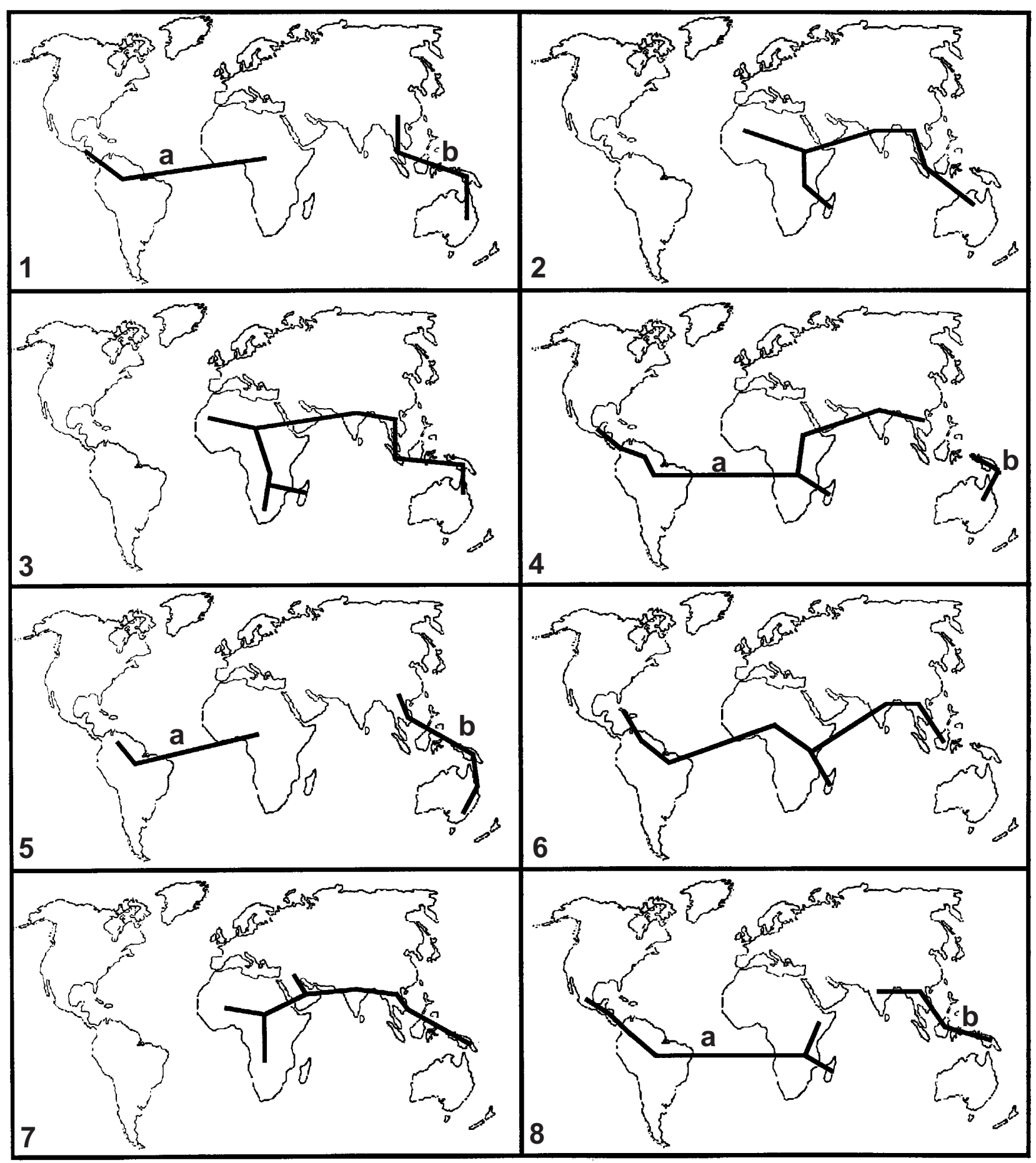

Figuras 1-8. Trazos individuales de géneros de Euphorbiaceae. 1. (a) Amanoa; (b) Actephila; 2. Cleistanthus; 3. Bridelia; 4. (a) Meineckia; (b) Choriceras; 5. (a) Pogonophora; (b) Austrobuxus; 6. Chaetocarpus; 7. Microdesmis; 8. (a) Caperonia; (b) Agrostistachys. 
Pseudagrostistachys, Paranecepsia, Pycnocoma, Sphaerostylis y Tragiella. De los géneros endémicos, los más diversos son Ditaxis (60 especies), Leucocroton (30) y Pera (40). Como ejemplos de distribuciones disyuntas se hallan Agrostistachys (figura 8b), Caperonia (figura 8a), Chaetocarpus (figura 6), Microdesmis (figura 7) y Pogonophora (figura 5a).

La subfamilia Crotonoideae tiene 12 tribus, 74 géneros y 1,436 especies (Radcliffe-Smith, 2001). Es predominantemente tropical, con su mayor centro de diversificación en la región Afrotropical (36.5\% de los géneros). Entre los géneros más diversos se encuentran Croton, con más de 1,000 especies, y Jatropha, con 175 (Govaerts et al., 2000). El endemismo genérico alcanza $68.9 \%$, concentrado principalmente en la regiones Neotropical y Afrotropical, la primera con 19 géneros (Acidocroton, Anomalocalyx, Cunuria, Cnidoscolus, Ditta, Dodecastigma, Garcia, Glycydendron, Joannesia, Julocroton, Manihot, Micrandra, Micrandropsis, Moacroton, Ophellantha, Pausandra, Sagotia, Sandwithia y Vaupesia) y la segunda con 17 (Benoistia, Cavacoa, Cladogelonium, Crotonogyne, Cyrtogonone, Domohinea, Grossera, Klaineanthus, Leeuwenbergia, Manniophyton, Mildbraedia, Neobuoutonia, Neoholstia, Parapantadenia, Ricinodendron, Schinziophyton y Tannodia). Ejemplos de distribuciones disyuntas son los géneros Dalechampia (figura 9), Endospermum (figura 10) y Suregada (figura 11).

La subfamilia Euphorbioideae es la que presenta el mayor número de apomorfías. Consta de cinco tribus, 46 géneros y 1,810 especies (Radcliffe-Smith, 2001). Su mayor centro de diversificación se encuentra en la región Neotropical (55.2\% de los géneros). En esta subfamilia se encuentra el género más diverso, Euphorbia sensu stricto, el cual posee alrededor de 1,000 especies. Otros géneros con diversidad considerable son Chamaesyce (250 especies) y Sapium y Sebastiania (100 especies cada uno). Los géneros presentan $63.0 \%$ de endemismo, con las regiones Neotropical y Afrotropical con el mayor número: 20 en la primera (Actinostemon, Adenopeltis, Algernonia, Bonania, Colliguaja, Cubanthus, Dalembertia, Dendrocousinsia, Dendrothrix, Dichostemma, Grimmeodendron, Mabea, Nealchornea, Ophthalmoblapton, Pachystroma, Peudosenefeldera, Rhodothyrsus, Senefeldera, Senefelderopsis y Tretraplandra) y 12 en la segunda (Afrotrewia, Anomostachys, Anthostema, Chlamydojatropha, Conosapium, Elaeophorbia, Endadenium, Hamilcoa, Monadenium, Plagiostyles, Sclerocroton y Synadenium). Ejemplos de distribuciones disyuntas son los géneros Excoecaria (figura 14), Microstachys (figura 13), Pimelodendron (figura 12) y Shirakopsis (figura 15).

La familia Euphorbiaceae sensu lato se distribuye en 11 regiones biogeográficas y presenta su mayor diversificación en las zonas tropicales. La subfamilia más diversa es
Acalyphoideae, con $36.6 \%$ de los géneros de la familia y un endemismo total de alrededor de $74 \%$. La región Neotropical presenta el mayor número de géneros (35\%) y el endemismo genérico más acentuado $(68.1 \%)$. Dentro de ella se localizan dos áreas importantes por su endemismo: la subregión Amazónica (Morrone, 2000), con 16 géneros endémicos (Angostylis, Anomalocalyx, Astrococcus, Chonocentrum, Cunuria, Didymocistus, Hevea, Jablonskia, Micrandra, Micrandropsis, Nealchornea, Podocalyx, Pseudosenefeldera, Polyandra, Tetraplandra y Vaupesia), y las Grandes Antillas, con 11 géneros endémicos (Acidocroton, Acidoton, Chascotheca, Cubanthus, Ditta, Grimmeodendron, Lasiocroton, Leucocroton, Moacroton, Platygyna y Picrodendron, los últimos dos restringidos a Cuba). En la región Afrotropical el área con mayor número de endemismos es Madagascar, con 15 (Amyrea, Anomostachys, Benoistia, Blotia, Bossera, Cladogelonium, Conosapium, Leptonema, Lobanilia, Petalodiscus, Claoxylopsis, Parapantadenia, Sphaerostylis, Stachyandra y Voatamalo). Australia posee 15 géneros endémicos (Adriana, Amperea, Beyeria, Bertya, Caelebogyne, Calycopeplus, Dissiliaria, Hylandia, Monotaxis, Neoroepera, Pseudanthus, Ricinocarpos, Rockinghamia, Sankowskia y Whyanbeelia, seis de ellos confinados a Queensland). Nueva Caledonia, de la región Neoguineana, posee seis géneros endémicos (Bocquillonia, Cocconerion, Longetia, Myricanthe, Neoguillauminia y Scagea).

Análisis panbiogeográfico. $\mathrm{El}$ primer análisis permitió obtener 24 cladogramas, de 435 pasos, índice de consistencia de 0.779 e índice de retención de 0.623 . Con base en los clados sustentados por más de dos sinapomorfías en el cladograma de consenso estricto se delimitaron dos trazos generalizados. Un trazo generalizado (figura 16a) une las regiones Neotropical y Afrotropical; los trazos individuales que lo sustentan son los de Amanoa, Caperonia, Conceveiba, Manprounea, Pogonophora, Savia y Tetrorchidium. La línea de base de este trazo es el Océano Atlántico. El segundo trazo generalizado (figura 16b) une las regiones Australiana Tropical, Australiana Templada, Neoguineana y Oriental, sustentado por los trazos individuales de Actephila, Baloghia, Choriceras, Petalostigma y Sauropus. La región Oriental se relaciona con la Neoguineana, relación sustentada por los trazos individuales de Agrotistachys, Baccaurea, Elateriospermum, Homonoia, Koilodepas, Ptychopyxis, Spathiostemon y Syndyophyllum. Los trazos individuales que unen estas dos regiones con Australia son los de Austrobuxus, Dimorphocalyx, Fontainea, Trigonostemon y Wetria. La línea de base de este trazo es el Océano Índico.

Luego de eliminadas las sinapomorfías se realizó un segundo análisis en el que se obtuvieron 23 cladogramas de 378 pasos, índice de consistencia de 0.78 e índice de reten- 


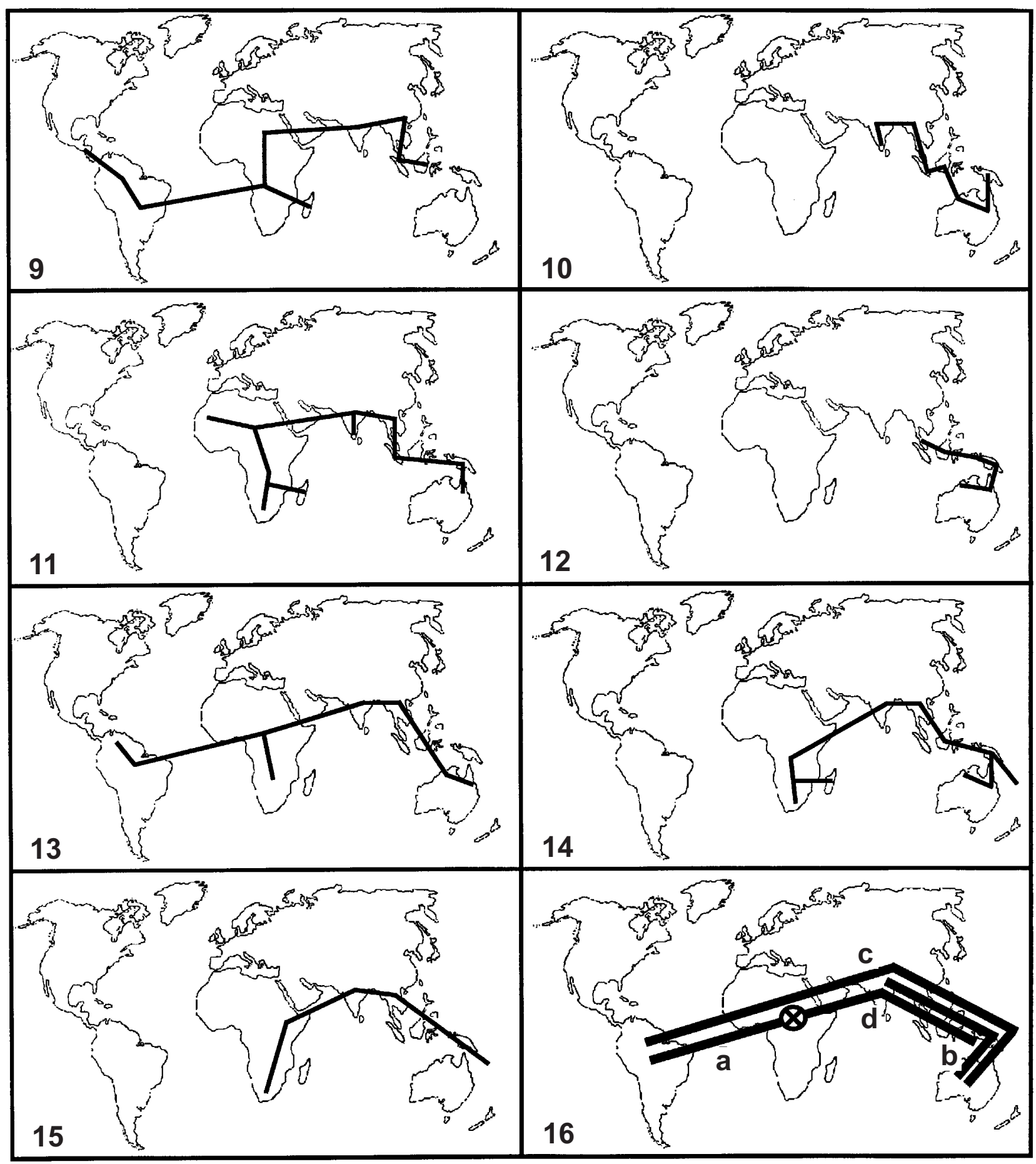

Figuras 9-16. Trazos individuales y generalizados de géneros de Euphorbiaceae. 9. Dalechampia; 10. Endospermum; 11. Suregada; 12. Pimelodendron; 13. Microstachys; 14. Excoecaria; 15. Shirakopsis; 16. trazos generalizados y nodo (señalado con un círculo con una " $\times$ " dentro). 
ción de 0.6. A partir de los clados sustentados por dos o más taxones, se identificaron dos trazos generalizados. El primero (figura 16c) une las regiones Australiana Templada, Australiana Tropical, Neoguineana, Afrotropical y Neotropical. Entre los trazos individuales que lo sustentan se encuentran los de Acalypha, Alchornea, Cleidion, Drypetes, Margaritaria, Microstachys, Omphalea y Phyllanthus. El otro trazo generalizado (figura 16d) une las regiones Neoguineana, Oriental y Afrotropical, y se encuentra sustentada por los trazos individuales de Glochidion, Macaranga, Microdesmis y Shirakopsis.

En un tercer análisis se eliminaron de nuevo las sinapomorfías, pero el cladograma de consenso estricto no tuvo resolución alguna.

Dos trazos generalizados se superponen en la región Afrotropical (figura 16), por lo que ésta es identificada como un nodo.

\section{Discusión y conclusiones}

En relación con la circunscripción de las Euphorbiaceae sensu lato, el análisis no brinda elementos para apoyar su separación en familias diferentes. Los trazos generalizados obtenidos poseen géneros de los distintos grupos analizados, no habiendo "sesgo" alguno en su composición.

De la distribución de los géneros se observa un desarrollo mayor en el reino Holotropical (Morrone, 2002), con centros de diversidad importantes en la región Afrotropical, principalmente en la franja que va de Nigeria y Camerún a Tanzania y Mozambique, y en Madagascar. Cabe destacar que la concentración de diversidad en estas áreas podría hacer pensar, desde una perspectiva dispersalista, en "centros de origen" o "áreas ancestrales"; sin embargo, la búsqueda de éstos está desechada por la perspectiva panbiogeográfica (Morrone y Crisci, 1995; Morrone, 2004). En la región Neotropical destaca la Amazonia; en la región Oriental, la zona de la Península Malaya; y en la Neoguineana, Nueva Guinea. Todas estas regiones pertenecen al reino Holotropical, excepto la última, dando sustento a la afirmación que se trata de una familia predominantemente gondwánica (Raven y Axelrod, 1974). A partir de los trazos generalizados obtenidos creemos que la evolución general de las Euphorbiaceae tuvo lugar en el supercontinente de Gondwana, siendo los principales eventos vicariantes involucrados los que llevaron a su fragmentación en los continentes del hemisferio sur.

Tomando en cuenta lo anterior y observando los principales patrones de distribución del hemisferio sur (SanMartín y Ronquist, 2004), puede verse que el primer trazo generalizado es congruente con el patrón gondwánico tropical (TPG), donde la región Afrotropical se encuentra cercanamente relacionada con la Neotropical, básicamente en la región norte de América del Sur (principalmente la Amazonia), donde se localiza uno de los centros de diver- sidad de la familia; además, se observa una relación cercana entre la flora de Madagascar y de África tropical (SanMartín y Ronquist, 2004). También puede observarse congruencia del segundo trazo generalizado, que une las regiones Australiana Templada y Tropical con las regiones Neoguineana y Oriental con el patrón gondwánico norte (NGP) de SanMartín y Ronquist (2004). Si bien estos autores han destacado la importancia de los eventos de dispersión para los grupos de plantas que analizaron, éstos no resultan aparentes en nuestro estudio, siendo el patrón básico vicariante.

Es importante puntualizar el alto grado de endemismo genérico regional que presenta la familia (61.3\%), lo que habla de una especiación activa y la escasa dispersión a otras regiones, después de los principales eventos vicariantes. Las regiones con mayor diversidad genérica son la Neotropical y la Afrotropical, seguidas de la región Oriental. De las tres, sólo las dos primeras tienen un endemismo alto $(68 \%$ y $55 \%$, respectivamente).

\section{Agradecimientos}

A Germán Carnevali Fernández Concha y un árbitro anónimo por sus útiles sugerencias.

\section{Literatura citada}

Airy Shaw H.K. 1971. The Euphorbiaceae of Siam. Kew Bulletin 26:241-251.

Airy Shaw H.K. 1980a. The Euphorbiaceae of New Guinea. Kew Bulletin 35:65-73.

Airy Shaw H.K.1980b. The Euphorbiaceae Platylobeae of Australia. Kew Bulletin 35:614-624.

Airy Shaw H.K. 1982. The Euphorbiaceae of Central Malesia (Celebes, Moluccas, Lesser Sunda Is.) Kew Bulletin 37:14-16.

Angiosperm Phylogeny Group (APG). 2003. An update of the Angiosperm Phylogeny Group classification for the orders and families of flowering plants: APGII. Botanical Journal of the Linnean Society 141:399-346.

Bentham G. 1878. Notes on Euphorbiaceae. Journal of the Proceedings of the Linnean Society. Botany 17:185-267.

Borhidi A. y Muñiz O. 1977. Revisión del género Croton L. (Euphorbiaceae) en Cuba. Annales Historico-Naturales Musei Nationalis Hungarici 69:41-53.

Craw R.C., Grehan J.R. y Heads M.J. 1999. Panbiogeography: Tracking the History of Life. Oxford Biogeography Series 11, Nueva York y Oxford.

Croizat L. 1940. Thirty-five new species of American Croton. Journal of the Arnold Arboretum 21:78-107.

Croizat L. 1941. Preliminaries for the study of Argentine and Uruguayan species of Croton. Darwiniana 5:417-462.

Croizat L. 1944. Additions to the genus Croton in South America. Darwiniana 6:442-468.

Croizat L. 1945. New or critical Euphorbiaceae from the Americas. Journal of the Arnold Arboretum 26:181-188.

Croizat L. 1958. Panbiogeography. Vol. 1, 2a, y 2b. Publicado por el autor, Caracas. 
Croizat L. 1964. Space, Time, Form: The Biological Synthesis. Publicado por el autor, Caracas.

Croizat L. 1972. An introduction to the subgeneric classification of Euphorbia L. with stress on the South African and Malagasy species. III. Webbia 27:1-221.

Escalante T. y Morrone J.J. 2003. ¿Para qué sirve el análisis de parsimonia de endemismos? En: Morrone J.J. y Llorente Bousquets J. Eds. Una Perspectiva Latinoamericana de la Biogeografía, pp. 167-172, Las Prensas de Ciencias, Universidad Nacional Autónoma de México, México, D.F.

García-Barros E., Gurrea P., Luciáñez M.J., Cano J.M., Munguira M.L., Moreno J.C., Sainz H., Sanza M.J. y Simón J.C. 2002. Parsimony analysis of endemicity and its application to animal and plant geographical distributions in the Ibero-Balearic region (western Mediterranean). Journal of Biogeography 29:109-124.

Goloboff P. 1999. NONA ver. 2.0. Publicado por el autor. Tucumán. Argentina. <http://www.cladistics.com>

Govaerts R., Frodin D.G. y Radcliffe-Smith A. 2000. World Checklist and Bibliography of Euphorbiaceae (and Pandanaceae). Vols. 1-4. The Royal Botanical Gardens, Kew.

Johnston M.C. 1959. The Texas species of Croton. Southwestern Naturalist 3:175-203.

Johnston M.C. y Warnock B.H. 1962. The ten species of Croton (Euphorbiaceae) occurring in far western Texas. Southwestern Naturalist 7:1-22.

Leach L.C. 1976. Distributional and morphological studies of the tribe Euphorbiae (Euphorbiaceae) and their classification and possible evolution. Excelsa 6:3-19.

Leandri J. 1939. Les Croton de Madagascar et des îles voisines. Annales de l'Institut Botanico-Geologique Colonial de Marseille (Serie 5) 7:1-100.

Leandri J. 1969. Sur quelques espèces malgaches de Croton (Euphorbiacées). Adansonia (Serie 2) 4:496-500.

Leandri J. 1971. Evolution morphologique récente des Croton malgaches. Adansonia (Serie 2) 11:41-46.

Leandri J. 1972a. Croton nouveaux de l'Ouest de Madagascar (Euphorbiacées). Adansonia (Serie 2) 12:65-71.

Leandri J. 1972b. Contribution a l'étude des Croton malgaches à grandes feuilles argentées. Adansonia (Serie 2) 12:403-408.

Luna-Vega I., Alcántara O., Espinosa-Organista D. y Morrone J.J. 1999. Historical relationships of the Mexican cloud forests: A preliminary vicariance model applying Parsimony Analysis of Endemicity to vascular plant taxa. Journal of Biogeography 26:1299-1305.

Luna-Vega I., Alcántara O., Morrone J.J. y Espinosa-Organista D. 2000. Track analysis and conservation priorities in the cloud forests of Hidalgo, Mexico. Diversity and Distributions 6:137143.

Lundell C.L. 1940. Noteworthy Spermatophytes from Mexico and Central America. Phytologia 1:449-451.

Mabberley D.J. 1998. The Plant-Book. Cambridge University Press, Cambridge.

McVaugh R. 1961. Euphorbiaceae novae novo-galicianae. Brittonia 13:145-205.

Martínez-Gordillo M. 1996. Contribución al Conocimiento del Género Croton (Euphorbiaceae) en el Estado de Guerrero, México. Contribuciones del Herbario de la Facultad de Ciencias No. 2, Universidad Nacional Autónoma de México, México, D.F.
Martínez-Gordillo M., Jiménez J., Cruz R., Juárez E., García R., Cervantes A. y Mejía R. 2002. Los géneros de la familia Euphorbiaceae en México. Anales del Instituto de Biología de la Universidad Nacional Autónoma de México, Serie Botánica 73:155-281.

Millspaugh C.F. 1916. Contributions to the North American Euphorbiaceae. Field Museum of Natural History, Botanical Series 2:401-420.

Morrone J.J. 1994a. On the identification of areas of endemism. Systematic Biology 43:438-441.

Morrone J.J. 1994b. Distributional patterns of species of Rhytirrhinini (Coleoptera: Curculionidae) and the historical relationships of the Andean provinces. Global Ecology and Biogeography Letters 4:188-194.

Morrone J.J. 1998. On Udvardy’s Insulantarctica province: A test from the weevils (Coleoptera: Curculionoidea). Journal of Biogeography 25:947-955.

Morrone J.J. 2000. A new regional biogeography of the Amazonian subregion, mainly based on animal taxa. Anales del Instituto de Biología, Universidad Nacional Autónoma de México, Serie Zoológica 71:99-123.

Morrone J.J. 2002. Biogeographic regions under track and cladistic scrutiny. Journal of Biogeography 29:149-152.

Morrone J.J. 2004. Homología Biogeográfica: Las Coordenadas Espaciales de la Vida. Instituto de Biología, Universidad Nacional Autónoma de México, México, D.F.

Morrone J.J. y Crisci J.V. 1995. Historical biogeography: Introduction to methods. Annual Review of Ecology and Systematics 26:373.401.

Morrone J.J. y J. Márquez. 2001. Halffter's Mexican Transition Zone, beetle generalised tracks, and geographical homology. Journal of Biogeography 28:635-650.

Müller J. 1865. Euphorbiaceae. Vorläufige Mitteilungen aus dem für De Candolle's Prodomus bestimmten Manuscript über diese Familie. Linnaea 43:1-224.

Müller J. 1866. Croton. En: de Candolle A.P. Ed. Prodromus Systematis Naturalis Regni Vegetabilis, 15(2). París, Estrasburgo y Londres.

Nixon K.C. 2002. WinClada ver. 1.00.08. Publicado por el autor. Ithaca, Nueva York. <http://www.cladistics.com>

Pax F. 1890. Euphorbiaceae. En: Engler A. Ed. Die Natürlichen Pflanzenfamilien, Erste Auflage, III 3:1-119. Leipzig.

Pax F. y Hoffman K. 1924. Die Phylogenie der Euphorbiaceae. Botanische Jahrbücher für Systematik, Pflanzengeschichte und Pflanzengeographie 59:129-182.

Pax F. y Hoffman K. 1931. Crotoneae. En: Engler A. y Prantl K. Eds. Die Natürlichen Pflanzenfamilien, Zweite Auflage, pp. 8388, Leipzig.

Radcliffe-Smith A.R. 1987. Flora of Tropical East Africa: Euphorbiaceae. A.A. Balkema, Rotterdam.

Radcliffe-Smith A. 2001. Genera Euphorbiacearum. Royal Botanic Gardens, Kew.

Raven P.H. y Axelrod D.I. 1974. Angiosperm biogeography and past continental movements. Annals of Missouri Botanical Garden 61:539-673.

Rosen B.R. 1988. From fossils to earth history: applied historical biogeography. En: Myers A.A. y Giller P.S. Eds. Analytical Biogeography, pp. 437-481, Chapman and Hall, Londres.

Rzedowski J. 1991. El endemismo de la flora fanerogámica mexicana: una apreciación análitica preliminar. Acta Botanica 
PATRONES DE ENDEMISMO Y DISYUNCIÓN DE GÉNEROS DE EUPHORBIACEAE

Mexicana 15:47-64.

SanMartín I. y Ronquist F. 2004. Southern hemisphere biogeography inferred by event-based models: Plant versus animal patterns. Systematic Biology 53:216-243.

Savolainen V., Fay M.F., Albach D.C., Backlund A., van der Bank M., Cameron K.M., Johnson S.A., Lledó M.D., Pintaud J., Powell M., Sheahan M.C., Soltis D.E., Soltis P.S., Weston P., Whitten W.M., Wurdack K.J. y Chase M.W. 2000. Phylogeny of the eudicots: a nearly complete familial analysis based on rbcL gene sequences. Kew Bulletin 55:257-309.

Soltis D.E., Soltis P.S., Chase M.W., Mort M.E., Albach D.C., Zanis M., Savolainen V., Hahn W.H., Hoot S.B., Fay M.F., Axtell M., Swensen S.M., Prince L.M., Kress W.J., Nixon K.C. y Farris J.S. 2000. Angiosperm phylogeny inferred from 18 S
rDNA, $r b c \mathrm{~L}$, and $a t p \mathrm{~B}$ sequences. Botanical Journal of the Linnaean Society 133:381-461.

Standley P.C. 1923.Trees and shrubs of Mexico. Contributions of the United States National Herbarium 23:610-620.

Standley P.C. y Steyermark J.A. 1949. Flora of Guatemala. Fieldiana: Botany 24:64-81.

Webster G.L. 1987. The saga of the spurges: a review of classification and relationships in the Euphorbiales. Botanical Journal of the Linnean Society 94:3-46.

Webster G.L. 1993. A provisional synopsis of the sections of the genus Croton (Euphorbiaceae). Taxon 42:793-823.

Webster G.L. 1994. Synopsis of the genera and suprageneric taxa of Euphorbiaceae. Annals of Missouri Botanical Garden 81:33-144.

Fecha de recepción: 2 de septiembre de 2004

Versión corregida: 24 de mayo de 2005

Aceptado: 8 de julio de 2005 
Apéndice 1. Matriz analizada, con el detalle de los géneros de Euphorbiaceae $s$. I. Los números junto a los géneros de la lista inferior corresponden a las columnas de la matriz, donde se indican de forma vertical.

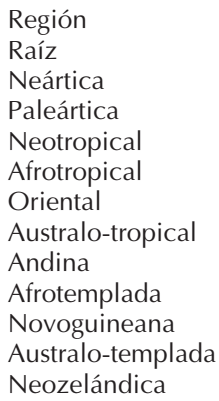

Continuación:
0000000000000000000000000000000000000000000000000000000000000000000000000000000000000 0000000001111111111222222222233333333334444444444555555555566666666667777777777888888 1234567890123456789012345678901234567890123456789012345678901234567890123456789012345

0000000000000000000000000000000000000000000000000000000000000000000000000000000000000 0000000000000000000000000000000000000000000000000000000000000010000000000000000000000 0000000000000001000000000000000000010000000000000000000000000000000000000000000000000 0110001010010011100100000000000000000000110000011001000100011101000000000000001110000 1101100001111100011111111011001111101111000100100110001011100000010000000000000001101 0000010000001101000100101111011101010000001001000010001010000000000000000000000000010 0000010000001101000000100001010100000000000000000010000000000000000000101000000000000 0000000000000000000000010000000000000000000000000000000000000000000000000000000000000 1000000100000000000000001000000000000000000000000000000000000000100000000000000000000 0000010000001101000000101011011101000000001011000010000010000000011100111111000000000 0000010000001101000000100010011101000000000000000010100000000000010111101001110000000 0000000000000000000000000000000000000000000000000000110000000000000000000000000000000

0000000000000011111111111111111111111111111111111111111111111111111111111111111111111 8888999999999900000000001111111111222222222233333333334444444444555555555566666666667 6789012345678901234567890123456789012345678901234567890123456789012345678901234567890

0000000000000000000000000000000000000000000000000000000000000000000000000000000000000 0000000000000000000000000000000000000100000000000000000000010000000000000000000000000 0000000000000000000000010000000001000000010000000001100000100000000000000000000000000 0001011000000000000000001110000000101100001000000000000000010111001010111000001010100 1111010000000101000110001000000001010011100111000000000111001000111000100100010010111 0010111011111110001001110000011111000000000000111111111001100000001001000001000111101 0000000000000000110000000000000000000000000000000000000000000000001000000010000000101 0000000000000000000000000000100000000000000000000000000000000000000000000000001000000 0010000000000000000000000000000000000000000000000000000000000000000000000000110000000 0000110111110110001001000000011011000000000000010110010000100000001000000110000110101 0000000000000000100000000000000000000000000000000000000000000000001100000010000110101 0000000000000000000000000000000000000000000000000000000000000000000000000000000000000

Continuación:

1111111111111111111111111111122222222222222222222222222222222222222222222222222222222 7777777778888888888999999999900000000001111111111222222222233333333334444444444555555 1123456789012345678901234567890123456789012345678901234567890123456789012345678901234

Región

Raíz

Neártica

Paleártica

Neotropical

Afrotropical

Oriental

Australo-tropical

Andina

Afrotemplada

Novoguineana

Australo-templada

Neozelándica

0000000000000000000000000000000000000000000000000000000000000000000000000000000000000 0000000000000001000000000000000000000000000000000000000000000000000000000000000000000 0000000000000000000000000011001000010000000000001000000000000010000000001000000000000 0000000000000001000111111100001111011111111101010000110001000001100000110010000000000 1111111110000001000000001000111000110000100011100110100000100000001001000000000000001 0010001001011001111000001011001000111000000000001101101110000010010101001101100000010 0000001000000000000000000000000000000000000000000100000000001000000001000000010110000 0000000000100000000000000000000000000000000000000000000000000000000000000000000000000 0000000000000000000000000000000000000000000000100000000000000000000000000000000000000 0000001000011111011000000011000000001000000000001101100000011110000111001101101001100 0000001000000101000000000000001000001000000000001000000000001100000111000000100000000 0000000000000000000000000000000000000000000000000000000000000000000000000000000000000 
Continuación: 2222222222222222222222222222222222222222222222233333333333333333333333333333333333333333333
555556666666666777777777788888888889999999999000000000111111111122222222223333333333
5678901234567890123456789012345678901234567890123456789012345678901234567890123456789

Región

Raíz

Neártica

Paleártica

Neotropical

Afrotropical

Oriental

Australo-tropical

Andina

Afrotemplada

Novoguineana

Australo-templada

Neozelándica
0000000000000000000000000000000000000000000000000000000000000000000000000000000000000 0011100000000000000000000000000000000000010011001000001100000000000000000101000000000 0010000000010000000000000000000010000000000000000011000001000000000000000101000000000 0110010000001000110000000000010100111011010011111000011000111111111110100101010001000 0010001111010110001111111110101011001100010010000100101010000001000011000111001111110 1010001001110001000000000001001010101000010110000011101000000000000010000101000000001 00100000000000000000000000000010101010000000000000000000000000000000000001101000000000 0010000000000000000000000000000000010000101000000000000000000000000000000101000000000 0010000000000000000000000001000001000000000000000000000000000000000000000101000000000 1010000001000001000000000001001010100000010010000010100000000000000000010101000000000 0010000000000000000000000001001010100000000000000000000000000000000000001101000000000 0010000000000000000000000000001000000000000000000000000000000000000000000101000000000
186. Acalypha L.

241. Acidocroton Griseb.

203. Acidoton Sw.

6. Actephila Blume

315. Actinostemon Mart. ex Klotzsch

145. Adelia L.

143. Adenochlaena Boivin ex Baill.

217. Adenocline Turcz.

296. Adenopeltis Bertero ex A.Juss.

128. Adenophaedra (Müll.Arg.) Müll.Arg.

160. Adriana Gaudich.

337. Afrotrewia Pax et K.Hoffm.

104. Agrostistachys Dalzell

152. Alchornea Sw.

122. Alchorneopsis Müll.Arg.

265. Aleurites J.R.Forst. et G.Forst.

321. Algernonia Baill.

249. Alphandia Baill.

12. Amanoa Aubl.

103. Amperea A.Juss.

125. Amyrea Leandri

24. Andrachne L.

86. Androstachys Prain

192. Angostylis Benth.

230. Annesijoa Pax et K.Hoffm.

272. Anomalocalyx Ducke

312. Anomostachys (Baill.) Hurus.

325. Anthostema A.Juss.

51. Antidesma L.

154. Aparisthmium Endl.

44. Apodiscus Hutch.

46. Aporosa Blume

131. Argomuellera Pax

113. Argythamnia P.Browne

83. Aristogeitonia Prain
45. Ashtonia Airy Shaw

15. Astrocasia B.L.Rob. et Millsp.

191. Astrococcus Benth.

66. Austrobuxus Miq.

181. Avellanita Phil.

43. Baccaurea Lour.

306. Balakata Esser

246. Baliospermum Blume

231. Baloghia Endl.

281. Benoistia H.Perrier et Leandri

123. Bernardia Houst. ex Mill.

251. Bertya Planch.

250. Beyeria Miq.

57. Bischofia Blume

243. Blachia Baill.

5. Blotia Leandri

132. Blumeodendron (Müll.Arg.) Kurz

155. Bocquillonia Baill.

295. Bonania A.Rich.

254. Borneodendron Airy Shaw

151. Bossera Leandri

135. Botryophora Hook.f.

31. Breynia J.R.Forst. et G.Forst.

14. Bridelia Willd.

153. Caelebogyne J.Sm.

328. Calycopeplus Planch.

67. Canaca Guillaumin

110. Caperonia A.St.-Hil.

120. Caryodendron H.Karst.

269. Cavacoa J.Léonard

49. Celianella Jabl.

58. Centroplacus Pierre

142. Cephalocroton Hochst.

141. Cephalocrotonopsis Pax

144. Cephalomappa Baill.

331. Chamaesyce Gray
91. Chaetocarpus Thwaites

17. Chascotheca Urb.

93. Cheilosa Blume

114. Chiropetalum A.Juss.

338. Chlamydojatropha Pax et K.Hoffm.

107. Chondrostylis Boerl.

9. Chonocentrum Pierre ex Pax et K.Hoffm.

71. Choriceras Baill.

119. Chrozophora Neck. ex A.Juss.

221. Cladogelonium Leandri

140. Cladogynos Zipp. ex Span.

170. Claoxylon A.Juss.

171. Claoxylopsis Leandri

138. Cleidiocarpon Airy Shaw

166. Cleidion Blume

13. Cleistanthus Hook.f. ex Planch.

88. Clutia L.

197. Cnesmone Blume

213. Cnidoscolus Pohl

180. Coccoceras Miq.

253. Cocconerion Baill.

240. Codiaeum Rumph. ex A.Juss.

291. Colliguaja Molina

156. Conceveiba Aubl.

293. Conosapium Müll.Arg.

179. Cordemoya Baill.

61. Croizatia Steyerm.

258. Croton L.

278. Crotonogyne Müll.Arg.

146. Crotonogynopsis Pax

259. Crotonopsis Michx.

332. Cubanthus (Boiss.) Millsp.

210. Cunuria Baill.

277. Cyrtogonone Prain 
106. Cyttaranthus J.Léonard

206. Dalechampia Plum. ex L.

318. Dalembertia Baill.

287. Dendrocousinsia Millsp.

302. Dendrothrix Esser

178. Deuteromallotus Pax et K.Hoffm.

226. Deutzianthus Gagnep.

326. Dichostemma Pierre

98. Dicoelia Benth.

56. Didymocistus Kuhlm.

238. Dimorphocalyx Thwaites

7. Discocarpus Klotzsch

172. Discoclaoxylon (Müll.Arg.) Pax et K.Hoffm.

127. Discocleidion (Müll.Arg.) Pax et K.Hoffm.

121. Discoglypremna Prain

68. Dissiliaria F.Muell. ex Baill.

112. Ditaxis Vahl ex A.Juss.

311. Ditrysinia Raf.

218. Ditta Griseb.

235. Dodecastigma Ducke

276. Domohinea Leandri

115. Doryxylon Zoll.

130. Droceloncia J.Léonard

34. Drypetes Vahl

164. Dysopsis Baill.

330. Elaeophorbia Stapf

222. Elateriospermum Blume

194. Eleutherostigma Pax et K.Hoffm.

335. Endadenium L.C.Leach

219. Endospermum Benth.

147. Enriquebeltrania Rzed.

136. Epiprinus Griff.

260. Eremocarpus Benth.

95. Erismanthus Wall. ex Müll.Arg.

169. Erythrococca Benth.

329. Euphorbia L.

288. Excoecaria L.

299. Falconeria Royle

25. Flueggea Willd.

239. Fontainea Heckel

100. Galearia Zoll. et Moritzi

268. Garcia Vahl ex Rohr

204. Gitara Pax et K.Hoffm.

157. Gavarretia Baill.

262. Givotia Griff.

32. Glochidion J.R.Forst. et G.Forst.

214. Glycydendron Ducke

3. Gonatogyne Klotzsch ex Müll.Arg.

294. Grimmeodendron Urb.

270. Grossera Pax

310. Gymnanthes Sw.
190. Haematostemon (Müll.Arg.) Pax et K.Hoffm.

284. Hamilcoa Prain

211. Hevea Aubl.

1. Heywoodia T.R.Sim

52. Hieronyma Allemão

301. Hippomane L.

286. Homalanthus A.Juss.

189. Homonoia Lour.

324. Hura L.

65. Hyaenanche Lamb.

232. Hylandia Airy Shaw

55. Hymenocardia Wall. ex Lindl.

42. Jablonskia G.L.Webster

223. Jatropha L.

228. Joannesia Vell.

261. Julocroton Mart.

74. Kairothamnus Airy Shaw

22. Keayodendron Leandri

215. Klaineanthus Pierre ex Prain

139. Koilodepas Hassk.

8. Lachnostylis Turcz.

187. Lasiococca Hook.f.

148. Lasiocroton Griseb.

229. Leeuwenbergia Letouzey et N.Hallé

163. Leidesia Müll.Arg.

50. Leptonema A.Juss.

16. Leptopus Decne.

149. Leucocroton Griseb.

33. Lingelsheimia Pax

174. Lobanilia Radcl.-Sm.

227. Loerzingia Airy Shaw

72. Longetia Baill.

309. Mabea Aubl.

168. Macaranga Thouars

40. Maesobotrya Benth.

177. Mallotus Lour.

212. Manihot Mill.

279. Manniophyton Müll.Arg.

319. Manprounea Aubl.

175. Mareya Baill.

176. Mareyopsis Pax et K.Hoffm.

27. Margaritaria L.f.

59. Martretia Beille

198. Megistostigma Hook.f.

20. Meineckia Baill.

118. Melanolepis Rchb.f. et Zoll.

161. Mercurialis L.

208. Micrandra Benth.

209. Micrandropsis Rodr.

77. Micrantheum Desf.

173. Micrococca Benth.

99. Microdesmis Hook.f. ex Hook.
292. Microstachys A.Juss.

255. Mildbraedia Pax

84. Mischodon Thwaites

257. Moacroton Croizat

333. Monadenium Pax

102. Monotaxis Brongn.

96. Moultonianthus Merr.

339. Myladenia Airy Shaw

252. Myricanthe Airy Shaw

285. Nealchornea Huber

124. Necepsia Prain

280. Neobuoutonia Müll.Arg.

327. Neoguillauminia Croizat

275. Neoholstia Rauschert

76. Neoroepera Müll.Arg. et F.Muell.

94. Neoscortechinia Hook.f. ex Pax

313. Neoshirakia Esser

183. Neotrewia Pax et K.Hoffm.

185. Octospermum Airy Shaw

82. Oldfieldia Benth. et Hook.f.

225. Oligoceras Gagnep.

207. Omphalea L.

242. Ophellantha Standl.

323. Ophthalmoblapton Allemão

54. Oreoporanthera Hutch.

150. Orfilea Baill.

233. Ostodes Blume

320. Pachystroma Müll.Arg.

205. Pachystylidium Pax et K.Hoffm.

101. Panda Pierre

236. Pantadenia Gagnep.

256. Paracroton Miq.

64. Paradrypetes Kuhlm.

126. Paranecepsia Radcl.-Sm.

237. Parapantadenia Capuron

80. Parodiodendron Hunz.

234. Pausandra Radlk.

336. Pedilanthus Neck. ex Poit.

11. Pentabrachion Müll.Arg.

92. Pera Mutis

4. Petalodiscus (Baill.) Pax

73. Petalostigma F.Muell.

111. Philyra Klotzsch

48. Phyllanoa Croizat

28. Phyllanthus L.

81. Picrodendron Griseb.

283. Pimelodendron Hassk.

79. Piranhea Baill.

282. Plagiostyles Pierre

202. Platygyna P.Mercier

304. Pleradenophora Esser

195. Plukenetia L.

133. Podadenia Thwaites

62. Podocalyx Klotzsch 
89. Pogonophora Miers ex Benth.

158. Polyandra Leal

53. Poranthera Rudge

39. Protomegabaria Hutch.

105. Pseudagrostistachys Pax et K.Hoffm.

78. Pseudanthus Sieber ex Spreng.

21. Pseudolachnostylis Pax

314. Pseudosenefeldera Esser

134. Ptychopyxis Miq.

36. Putranjiva Wall.

129. Pycnocoma Benth.

266. Reutealis Airy Shaw

29. Reverchonia A.Gray

317. Rhodothyrsus Esser

41. Richeria Vahl

26. Richeriella Pax et K.Hoffm.

248. Ricinocarpos Desf.

263. Ricinodendron Müll.Arg.

159. Ricinus L.

184. Rockinghamia Airy Shaw

193. Romanoa Trevis.

245. Sagotia Baill.

167. Sampantaea Airy Shaw

273. Sandwithia Lanj.

69. Sankowskia P.I.Forst.

300. Sapium Jacq.

30. Sauropus Blume
2. Savia Willd.

75. Scagea McPherson

264. Schinziophyton Hutch. ex Radcl.-Sm.

305. Sclerocroton Hochst.

290. Sebastiania Spreng.

23. Securinega Comm. ex Juss.

162. Seidelia Baill.

316. Senefeldera Mart.

303. Senefelderopsis Steyerm.

308. Shirakopsis Esser

35. Sibangea Oliv.

188. Spathiostemon Blume

298. Spegazziniophytum Esser

109. Speranskia Baill.

199. Sphaerostylis Baill.

108. Sphyranthera Hook.f.

289. Spirostachys Sond.

37. Spondianthus Engl.

87. Stachyandra J.-F.Leroy ex Radcl.-Sm.

297. Stillingia Garden ex L.

244. Strophioblachia Boerl.

116. Sumbaviopsis J.J.Sm.

220. Suregada Roxb. ex Rottler 137. Symphyllia Baill.

334. Synadenium Boiss.
97. Syndyophyllum K.Schum. et Lauterb.

60. Tacarcuna Huft

274. Tannodia Baill.

271. Tapoides Airy Shaw

63. Tetracoccus Engelm. ex Parry

322. Tetraplandra Baill.

216. Tetrorchidium Poepp.

47. Thecacoris A.Juss.

117. Thyrsanthera Pierre ex Gagnep.

201. Tragia Plum. ex L.

200. Tragiella Pax et K.Hoffm.

182. Trewia L.

307. Triadica Lour.

90. Trigonopleura Hook.f.

247. Trigonostemon Blume

38. Uapaca Baill.

224. Vaupesia R.E.Schult.

267. Vernicia Lour.

196. Vigia Vell.

85. Voatamalo Capuron ex Bosser

165. Wetria Baill.

70. Whyanbeelia Airy Shaw et B.Hyland

10. Wielandia Baill.

18. Zimmermannia Pax

19. Zimmermanniopsis Radcl.-Sm. 